PROCEEDINGS OF THE

AMERICAN MATHEMATICAL SOCIETY

Volume 138, Number 6, June 2010, Pages 1965-1968

S 0002-9939(10)10235-4

Article electronically published on February 12, 2010

\title{
ON THE FINITENESS OF ASSOCIATED PRIMES OF LOCAL COHOMOLOGY MODULES
}

\author{
PHAM HUNG QUY
}

(Communicated by Bernd Ulrich)

\begin{abstract}
Let $R$ be a Noetherian ring, $\mathfrak{a}$ be an ideal of $R$ and $M$ be a finitely generated $R$-module. The aim of this paper is to show that if $t$ is the least integer such that neither $H_{\mathfrak{a}}^{t}(M) \operatorname{nor} \operatorname{supp}\left(H_{\mathfrak{a}}^{t}(M)\right)$ is non-finite, then $H_{\mathfrak{a}}^{t}(M)$ has finitely many associated primes. This combines the main results of Brodmann and Faghani and independently of Khashyarmanesh and Salarian.
\end{abstract}

\section{INTRODUCTION}

Throughout this paper, $R$ is a Noetherian ring (with identity), $\mathfrak{a}$ is an ideal of $R$ and $M$ is an $R$-module. For basic facts about commutative algebra see 3 and [8]; for local cohomology refer to [2]. A module is finite if it is finitely generated and a set is finite if it has finitely many elements. We use $\mathbb{N}_{0}$ to denote the set of non-negative integers.

An interesting problem in commutative algebra is determining when the set of associated primes of the $i$ th local cohomology module $H_{\mathfrak{a}}^{i}(M)$ of $M$ is finite. If $R$ is a regular local ring containing a field, then $H_{\mathfrak{a}}^{t}(R)$ has only finitely many associated primes for all $i \geq 0$; cf. 4] (in positive characteristic) and 7] (in characteristic zero). However, Katzman [5] has given an example of a Noetherian local ring and an ideal $\mathfrak{a}$ such that $H_{\mathfrak{a}}^{2}(R)$ has infinitely many associated primes. But we have many interesting results about the finiteness of $\operatorname{Ass}_{R}\left(H_{\mathfrak{a}}^{t}(M)\right)$. It is well known that if $M$ is finite, then $\operatorname{Ass}_{R}\left(H_{\mathfrak{a}}^{t}(M)\right)$ is finite in either of the following cases:

(a) $H_{\mathfrak{a}}^{i}(M)$ is finite for all $i<t$; see [1] and [6];

(b) $\operatorname{supp}\left(H_{\mathfrak{a}}^{t}(M)\right)$ is finite for all $i<t$; see [6].

The aim of this paper is to combine (a) and (b). That is, if $M$ is finitely generated, then $H_{\mathfrak{a}}^{t}(M)$ has only finitely many associated primes if, for all $i<t$, $H_{\mathfrak{a}}^{i}(M)$ is finite or has finite support.

In section 2, we define: $M$ is an FSF module if there is a finite submodule $N$ of $M$ such that the quotient module $M / N$ has finite support, and we give some properties of FSF modules.

In section 3, we will prove the following: Let $\mathfrak{a}$ be an ideal of the Noetherian ring $R$, and let $M$ be an FSF $R$-module. Let $t \in \mathbb{N}_{0}$ be such that $H_{\mathfrak{a}}^{i}(M)$ is FSF for all $i<t$. Then $\operatorname{Hom}_{R}\left(R / \mathfrak{a}, H_{\mathfrak{a}}^{t}(M)\right)$ is FSF. Therefore, $\operatorname{Ass}_{R}\left(H_{\mathfrak{a}}^{t}(M)\right)$ is finite. This implies the main result as a consequence.

Received by the editors March 23, 2009, and, in revised form, October 1, 2009.

2010 Mathematics Subject Classification. Primary 13D45, 13E99.

Key words and phrases. Local cohomology, associated primes.

(c) 2010 American Mathematical Society Reverts to public domain 28 years from publication 


\section{FSF MODULE}

Definition 2.1. Let $R$ be a Noetherian ring and $M$ be an $R$-module. $M$ is called an FSF module if there is a Finite submodule $N$ of $M$ such that Support of the quotient module $M / N$ is Finite.

Proposition 2.2. Let $M$ be an $R$-module. We have

(i) If $M$ is an FSF module, then $\operatorname{Ass}_{R}(M)$ is finite.

(ii) Let $0 \rightarrow M_{1} \rightarrow M \rightarrow M_{2} \rightarrow 0$ be an exact sequence of $R$-modules. Then $M$ is FSF iff $M_{1}$ and $M_{2}$ are FSF. $i \geq 0$.

(iii) Let $M$ be an FSF module and $N$ be finite. Then $\operatorname{Ext}_{R}^{i}(N, M)$ is FSF for all

Proof. (i). This is trivial from the definition of FSF modules.

(ii). " $\Rightarrow$." If $M$ is an FSF module, it is easy to show that $M_{1}$ and $M_{2}$ are FSF.

" $\Leftarrow$." Suppose that $M_{1}$ and $M_{2}$ are FSF. Let $N_{1}$ and $N_{2}$ be finitely generated submodules of $M_{1}$ and $M_{2}$, respectively, such that $\operatorname{supp}\left(M_{1} / N_{1}\right)$ and $\operatorname{supp}\left(M_{2} / N_{2}\right)$ are finite. We may assume that $M_{1}$ is a submodule of $M$ and that $M_{2}$ is a quotient module of $M$. Let $x_{1}, x_{2}, \ldots, x_{n}, y_{1}, y_{2}, \ldots, y_{m}$ in $M$ such that $x_{1}, x_{2}, \ldots, x_{n}$ are generators of $N_{1}$ and $\bar{y}_{1}, \bar{y}_{2}, \ldots, \bar{y}_{m}$ are generators of $N_{2}$ in $M_{2}=M / M_{1}$. Let $N$ be a submodule of $M$ generated by $x_{1}, x_{2}, \ldots, x_{n}, y_{1}, y_{2}, \ldots, y_{m}$, so $N$ is finite, and it is not difficult to show that $\operatorname{supp}(M / N)$ is finite. Hence, $M$ is FSF.

(iii) $M$ is FSF, so there exists an exact sequence

$$
0 \longrightarrow M_{1} \longrightarrow M \longrightarrow M_{2} \longrightarrow 0,
$$

with $M_{1}$ finitely generated and $\operatorname{supp}\left(M_{2}\right)$ finite. This exact sequence induces exact sequences

$$
\operatorname{Ext}_{R}^{i}\left(N, M_{1}\right) \longrightarrow \operatorname{Ext}_{R}^{i}(N, M) \longrightarrow \operatorname{Ext}_{R}^{i}\left(N, M_{2}\right)
$$

for all $i \in \mathbb{N}_{0}$. Since $N$ and $M_{1}$ are finitely generated modules and $\operatorname{supp}\left(M_{2}\right)$ is finite, we have that $\operatorname{Ext}_{R}^{i}\left(N, M_{1}\right)$ is finitely generated and $\operatorname{supp}\left(\operatorname{Ext}_{R}^{i}\left(N, M_{2}\right)\right)$ is finite. Hence, $\operatorname{Ext}_{R}^{i}(N, M)$ is FSF for all $i \in \mathbb{N}_{0}$.

\section{THE MAIN RESUlT}

Proposition 3.1. Let $\mathfrak{a}$ be an ideal of the Noetherian ring $R$, and let $M$ be an FSF $R$-module. Let $t \in \mathbb{N}_{0}$ be such that $H_{\mathfrak{a}}^{i}(M)$ is FSF for all $i<t$. Then

$$
\operatorname{Hom}_{R}\left(R / \mathfrak{a}, H_{\mathfrak{a}}^{t}(M)\right)
$$

is FSF. Therefore, $\operatorname{Ass}_{R}\left(H_{\mathfrak{a}}^{t}(M)\right)$ is finite.

Proof. The last assertion follows from the first, from Proposition 2.2(i) and from the fact that $\operatorname{Ass}_{R}\left(H_{\mathfrak{a}}^{t}(M)\right)=\operatorname{Ass}_{R}\left(\operatorname{Hom}\left(R / \mathfrak{a}, H_{\mathfrak{a}}^{t}(M)\right)\right)$.

We prove that $\operatorname{Hom}_{R}\left(R / \mathfrak{a}, H_{\mathfrak{a}}^{t}(M)\right)$ is FSF by induction on $t$. The case $t=0$ is clear because $\operatorname{Hom}_{R}\left(R / \mathfrak{a}, H_{\mathfrak{a}}^{0}(M)\right) \subseteq M$.

So, let $t>0$ and set $\bar{M}=M / H_{\mathfrak{a}}^{0}(M)$. Then $\bar{M}$ is FSF, $H_{\mathfrak{a}}^{0}(\bar{M})=0$, and

$$
H_{\mathfrak{a}}^{k}(\bar{M}) \cong H_{\mathfrak{a}}^{k}(M)
$$

for all $k>0$. Thus $H_{\mathfrak{a}}^{i}(\bar{M})$ is FSF for all $i<t$ and $H_{\mathfrak{a}}^{t}(\bar{M}) \cong H_{\mathfrak{a}}^{t}(M)$. Replace $M$ by $\bar{M}$ and assume henceforth that $H_{\mathfrak{a}}^{0}(M)=0$. By Proposition 2.2(i), we have that $\operatorname{Ass}_{R}(M)$ is finite. Combining this with $H_{\mathfrak{a}}^{0}(M)=0$ implies that there exists $a \in \mathfrak{a}$ such that $a$ is an $M$-regular element. So, we have the short exact sequence

$$
0 \longrightarrow M \stackrel{a \cdot}{\longrightarrow} M \stackrel{p}{\longrightarrow} M / a M \longrightarrow 0,
$$


where $p$ is natural projection. This yields the exact cohomology sequences

$$
H_{\mathfrak{a}}^{i}(M) \longrightarrow H_{\mathfrak{a}}^{i}(M / a M) \longrightarrow H_{\mathfrak{a}}^{i+1}(M) \quad\left(\forall i \in \mathbb{N}_{0}\right) .
$$

Hence, $H_{\mathfrak{a}}^{i}(M / a M)$ is FSF for all $i<t-1$. It is clear that $M / a M$ is FSF, so by induction, we have that $\operatorname{Hom}_{R}\left(R / \mathfrak{a}, H_{\mathfrak{a}}^{t-1}(M / a M)\right)$ is FSF.

We consider the long exact sequence

$$
H_{\mathfrak{a}}^{t-1}(M) \stackrel{a \cdot}{\longrightarrow} H_{\mathfrak{a}}^{t-1}(M) \stackrel{H_{\mathfrak{a}}^{t-1}(p)}{\longrightarrow} H_{\mathfrak{a}}^{t-1}(M / a M) \longrightarrow H_{\mathfrak{a}}^{t}(M) \stackrel{a \cdot}{\longrightarrow} H_{\mathfrak{a}}^{t}(M) .
$$

Let $N=\frac{H_{\mathfrak{a}}^{t-1}(M)}{a H_{\mathfrak{a}}^{t-1}(M)}$ and $N^{\prime}=\operatorname{coker}\left(H_{\mathfrak{a}}^{t-1}(p)\right)$. We split the exact sequence $(*)$ into two exact sequences:

$$
\begin{gathered}
0 \longrightarrow N \longrightarrow H_{\mathfrak{a}}^{t-1}(M / a M) \longrightarrow N^{\prime} \longrightarrow 0, \\
0 \longrightarrow N^{\prime} \longrightarrow H_{\mathfrak{a}}^{t}(M) \stackrel{a .}{\longrightarrow} H_{\mathfrak{a}}^{t}(M) .
\end{gathered}
$$

From sequence $(* *)$ we deduce that the sequence

$$
\operatorname{Hom}_{R}\left(R / \mathfrak{a}, H_{\mathfrak{a}}^{t-1}(M / a M)\right) \longrightarrow \operatorname{Hom}_{R}\left(R / \mathfrak{a}, N^{\prime}\right) \longrightarrow \operatorname{Ext}_{R}^{1}(R / \mathfrak{a}, N)
$$

is exact. The left-most module is FSF as above and the right-most module is FSF by Proposition 2.2(iii); therefore, $\operatorname{Hom}_{R}\left(R / \mathfrak{a}, N^{\prime}\right)$ is FSF. Furthermore, $(* * *)$ gives the exact sequence

$$
0 \longrightarrow \operatorname{Hom}_{R}\left(R / \mathfrak{a}, N^{\prime}\right) \longrightarrow \operatorname{Hom}_{R}\left(R / \mathfrak{a}, H_{\mathfrak{a}}^{t}(M)\right) \stackrel{a \cdot}{\longrightarrow} \operatorname{Hom}_{R}\left(R / \mathfrak{a}, H_{\mathfrak{a}}^{t}(M)\right) .
$$

On the other hand, the multiplication homomorphism

$$
a \cdot: \operatorname{Hom}_{R}\left(R / \mathfrak{a}, H_{\mathfrak{a}}^{t}(M)\right) \rightarrow \operatorname{Hom}_{R}\left(R / \mathfrak{a}, H_{\mathfrak{a}}^{t}(M)\right)
$$

is zero since $a \in \mathfrak{a}$.

So, we have that $\operatorname{Hom}_{R}\left(R / \mathfrak{a}, H_{\mathfrak{a}}^{t}(M)\right) \cong \operatorname{Hom}_{R}\left(R / \mathfrak{a}, N^{\prime}\right)$ is FSF, as desired.

Finally, we have

Theorem 3.2. Let $\mathfrak{a}$ be an ideal of the Noetherian ring $R$, and let $M$ be a finitely generated $R$-module. Let $t \in \mathbb{N}_{0}$ be such that either $H_{\mathfrak{a}}^{i}(M)$ is finite or $\operatorname{supp}\left(H_{\mathfrak{a}}^{i}(M)\right)$ is finite for all $i<t$. Then $\operatorname{Ass}_{R}\left(H_{\mathfrak{a}}^{t}(M)\right)$ is finite.

\section{REFERENCES}

[1] M. Brodmann, A. L. Faghani, A finiteness result for associated primes of local cohomology modules, Proc. Amer. Math. Soc., 128(2000), 2851-2853. MR.1664309 (2000m:13028)

[2] M. Brodmann, R. Y. Sharp, Local cohomology: An algebraic introduction with geometric applications, Cambridge University Press, 1998. MR.1613627 (99h:13020)

[3] W. Bruns, J. Herzog, Cohen-Macaulay rings, Cambridge University Press, 1993. MR.1251956 (95h:13020)

[4] C. Huneke, R. Sharp, Bass numbers of local cohomology modules, Trans. Amer. Math. Soc., 339 (1993), 765-779. MR1124167 (93m:13008)

[5] M. Katzman, An example of an infinite set of associated primes of a local cohomology module, J. Algebra, 252 (2002), no. 1, 161-166. MR1922391(2003h:13021)

[6] K. Khashyarmanesh, Sh. Salarian, On the associated primes of local cohomology modules, Comm. Algebra, 27(1999), 6191-6198. MR1726302 (2000m:13029) 
[7] G. Lyubeznik, Finiteness properties of local cohomology modules (an application of $D$-modules to commutative algebra). Invent. Math, 113 (1993), 41-55. MR.1223223 (94e:13032)

[8] H. Matsumura, Commutative ring theory, Cambridge University Press, 1986. MR.879273 (88h:13001)

Department of Mathematics, FPt University (Dai Hoc FPt), 15B Pham Hung Street, Ha Noi, Vietnam

E-mail address: phamhungquy@gmail.com

E-mail address: quyph@fpt.edu.vn 